\title{
HUMANIZAÇÃO DA ASSISTÊNCIA DE ENFERMAGEM EM UNIDADE DE TERAPIA INTENSIVA NEONATAL
}

\section{HUMANIZATION OF NURSING ASSISTANCE IN A NEONATAL INTENSIVE THERAPY UNIT}

\section{HUMANIZACIÓN DE LA ATENCIÓN DE ENFERMERÍA EN UNA UNIDAD DE TERAPIA INTENSIVA NEONATAL}

Pamela Iasmine Amorim Garcia Leite ${ }^{1}$, Fabricio da Gama Pereira ${ }^{2}$, Rafael Fernandes Demarchi ${ }^{3}$, Thalise Yuri Hattori ${ }^{4}$, Vagner Ferreira do Nascimento ${ }^{5}$, Ana Claudia Pereira Terças-Trettel ${ }^{6}$

\begin{abstract}
RESUMO
Objetivo: compreender a humanização da assistência de enfermagem em unidade de terapia intensiva neonatal de hospital privado mato-grossense. Método: utilizou-se como percurso metodológico o estudo de caráter descritivo, exploratório e qualitativo sob o parecer ético em pesquisa 1.033.746/2015. A coleta de dados foi realizada em 2016 com 4 enfermeiras seguida por análise de conteúdo. Resultados: Os profissionais demonstram grande conhecimento sobre a humanização, compreendendo a mesma como um processo vivencial, que é adquirido por intermédio da experiência da prática clínica sob um viés afetivo e da própria ciência da enfermagem. Destacou-se a importância da sua prática na prestação do cuidado de enfermagem ao neonato, devendo a atenção humanizada ser estendida à família do paciente hospitalizado. Conclusão: Percebeu-se uma preocupação dos profissionais, em relação à importância do envolvimento familiar no processo de humanização, que perpassa pela confiança mútua até o processo de empoderamento gerado nos pais pela equipe.
\end{abstract}

Descritores: Humanização da Assistência; Enfermagem; Unidades de Terapia Intensiva Neonatal.

\begin{abstract}
Objective: to understand the humanization of nursing care in the neonatal intensive care unit of a private hospital in Mato Grosso. Method: a descriptive, exploratory and qualitative study was used as methodological course under the ethical opinion in research 1,033,746/2015. Data collection was performed in 2016 with 4 nurses followed by content analysis. Results: The professionals demonstrate a great knowledge about humanization, understanding it as a experiential process, which is acquired through the experience of clinical practice under an affective bias and the very science of nursing. It was emphasized the importance of its practice in the provision of nursing care to the neonate, and the humanized care should be extended to the family of the hospitalized patient. Conclusion: It was noticed a concern of the professionals, regarding the importance of the family involvement in the process of humanization, that crosses by the mutual confidence until the process of empowerment generated in the parents by the team.

Descriptors: Humanization of Assistance; Nursing; Neonatal Intensive Care Units.

\footnotetext{
${ }^{1}$ Enfermeira egressa da Universidade do Estado de Mato Grosso/UNEMAT.

${ }^{2}$ Acadêmico de Enfermagem da Universidade do Estado de Mato Grosso.

${ }^{3}$ Enfermeiro egresso da Universidade do Estado de Mato Grosso/UNEMAT.

${ }^{4}$ Enfermeira, Professora Mestre, Universidade do Estado de Mato Grosso/UNEMAT.

${ }^{5}$ Enfermeiro, Professor Doutor, Universidade do Estado de Mato Grosso/UNEMAT.

${ }^{6}$ Docente adjunta UNEMAT, departamento de Enfermagem. Docente permanente do Mestrado em Saúde Coletiva da UFMT.
} 


\section{RESUMEN}

Objetivo: Buscando comprender la humanización de la atención de enfermería en una unidad de cuidados intensivos neonatales de un hospital privado en Mato Grosso. Método: el estudio descriptivo, exploratorio y cualitativo se utilizó como un enfoque metodológico bajo la opinión ética en la investigación 1.033.746/2015. La recopilación de datos se realizó en 2016 con 4 enfermeras seguidas de análisis de contenido. Resultados: Los profesionales demuestran un gran conocimiento sobre la humanización, entendiéndolo como un proceso experimental, que se adquiere a través de la experiencia de la práctica clínica bajo un sesgo afectivo y la ciencia de la enfermería misma. Se destacó la importancia de su práctica de proporcionar cuidados de enfermería al neonato, y la atención humanizada debería extenderse a la familia del paciente hospitalizado. Conclusión: Se observó una preocupación de los profesionales, con respecto a la importancia de la participación familiar en el proceso de humanización, que pasa por la confianza mutua hasta el proceso de empoderamiento generado por el equipo en los padres.

Descriptores: Humanización de la asistencia; Enfermería; Unidades de cuidados intensivos neonatales.

\section{INTRODUÇÃO}

A humanização em saúde no Brasil, no tocante à saúde da criança e do adolescente, inicia-se no aspecto jurídico, por meio do Estatuto da Criança e do Adolescente (ECA), Lei nº 8.069 de 1990, onde se estabeleceu os direitos de proteção à saúde e à vida, além da obrigatoriedade dos serviços de saúde manterem condições para que menores de idade, durante o período de internação, possam permanecer na companhia de seus pais ou responsável legal. ${ }^{1}$ Com os avanços nas discussões sobre essa temática, o Ministério da Saúde (MS) prorrogou por intermédio da Portaria nº 693 de 2000, o Método Mãe Canguru (MMC), que dispõe de diretrizes para atenção humanizada ao recém-nascido de baixo peso. ${ }^{2,3}$

Em 2001, surge o Programa Nacional de Humanização Hospitalar
(PNHAH), logo após, em 2003, iniciaram propostas para que o processo de humanização deixasse de ser restrito apenas ao ambiente hospitalar, mas sim uma política incorporada a todos os níveis de atenção à saúde do Sistema Único de Saúde (SUS). Deste modo o MS lançou a Política Nacional de Humanização (PNH) que assume o dever de diminuir a precarização do processo de trabalho em saúde e as influências negativas técnicas e burocráticas sob os trabalhadores e usuários dos serviços. ${ }^{4}$

Uma década após a publicação da PNH, constata-se que sua implantação nos serviços de saúde ainda é frágil, com a persistência dos problemas que incentivaram sua criação, estando ainda evidente a falta de compreensão desta política por parte dos profissionais, que ainda acreditam que a humanização se 
remete tão somente ao bom relacionamento entre companheiros de trabalho e ao cuidado cativo do profissional com o cliente. ${ }^{5,6}$

Porém, para pensar em humanização no âmbito hospitalar é necessário considerar as necessidades dos profissionais e clientes frente às condições estruturais e políticas da instituição, bem como entender a fragmentação do cuidado em saúde e o respeito aos princípios doutrinários do SUS. ${ }^{7}$ Para tanto, algumas clientelas parecem impor desafios extras à assistência humanizada, já que possuem dependências que exigem de seus familiares maior envolvimento e cuidado, como as crianças. Sendo assim, a hospitalização de uma criança, em especial no período neonatal constitui-se em um evento danoso ao binômio mãe-filho, podendo desencadear uma série de fragilidades. $\mathrm{O}$ medo, na ocasião, deve-se, primordialmente, à ideia de que o RN passará a pertencer aos profissionais e não aos pais. ${ }^{8,9}$

Nesse sentido, a humanização da assistência de enfermagem nos serviços de saúde, particularmente em Unidades de Terapia Intensiva Neonatal (UTIN), deve partir do princípio de que se o indivíduo é acolhido, suas queixas e angústias ouvidas ativamente e respeitado pela equipe multiprofissional seu tratamento será mais eficaz. ${ }^{6}$ Para isso, torna-se essencial o desenvolvimento do vínculo famíliaequipe, ao passo que essa relação fornece subsídios para a implementação da assistência humanizada. ${ }^{10,11}$

Neste contexto, humanizar nesse espaço de cuidado, também remete-se principalmente ao acolhimento e apoio às famílias, atentando-se àquelas que deixam seus compromissos e suas vidas em outros municípios e/ou regiões, deslocando-se em busca de maiores recursos em saúde em cidades polo, normalmente depois de peregrinações e angústia por atendimento imediato ao seu familiar que acaba de nascer. $^{12}$

Sendo assim, considerando a importância do cuidado humanizado e dos possíveis sofrimentos que podem ser atenuados ou prevenidos, este estudo objetivou compreender a percepção dos enfermeiros sobre $\mathrm{o}$ processo de humanização da assistência de enfermagem em UTIN.

\section{MÉTODO}

Para atender ao objetivo proposto realizou-se uma pesquisa de caráter descritivo, exploratório, seguindo uma abordagem qualitativa. $\mathrm{O}$ estudo foi realizado em uma UTIN localizada em uma 
instituição de saúde privada no município de Tangará da Serra na região Médio Norte do estado de Mato Grosso. A instituição possui 33 leitos para atendimento maternoinfantil e 12 leitos de UTIN. A escolha se deu devido tal estabelecimento de saúde ser o único de referência na região de saúde, atendendo mais de 10 municípios do estado de Mato Grosso.

A coleta de dados foi realizada entre abril e maio de 2016, através da aplicação de questionário semiestruturado elaborado pelos autores que continham questões socioeconômicas e relacionadas à humanização no serviço. Após consentimento, o instrumento de coleta de dados foi entregue em envelopes individuais aos participantes após esclarecimento de dúvidas, tendo o prazo de 48 horas para devolutiva. Após recolhimento dos questionários respondidos, os dados foram transcritos na íntegra, organizados de forma sistematizada, por meio de um sistema de classificação, com codificação do tipo alfanumérica, onde as consoantes $\mathrm{E}$ referem-se a enfermeiro, seguidas de número arábico que compôs o conjunto para determinar a sequência de realização das entrevistas.

Os dados foram analisados por meio da técnica de análise de conteúdo, que se constitui no conjunto de técnicas de análise das comunicações e utiliza procedimentos sistemáticos e objetivos de descrição do conteúdo das mensagens. ${ }^{13}$ Esta técnica é composta por três etapas: pré-análise, exploração do material, tratamento e interpretação dos resultados. A partir de então, emergiram três núcleos de sentidos agrupados em categoria única sendo eles: conhecimento das enfermeiras sobre humanização, métodos de humanização em UTIN e dificuldades na implantação de alguns métodos de humanização.

Como critério de inclusão optou-se em entrevistar sujeitos com formação superior em enfermagem e que tiveram contato com o setor nos últimos 12 meses, além de obrigatoriamente estarem vinculados a este serviço de saúde e que aceitaram participar da pesquisa assinando o Termo de Consentimento Livre e Esclarecido (TCLE). Foram excluídos àqueles que estavam de atestados ou licenças no período da pesquisa. Todos os enfermeiros que atuam na instituição $(\mathrm{N}=10)$ foram contatados, destes quatro foram entrevistados pois definiu-se $o$ tamanho amostral ao identificar exaustividade das informações de interesse, como proposto pela saturação teórica. ${ }^{14}$

O estudo respeitou todos os aspectos éticos em pesquisa com seres humanos, em 
conformidade com a Resolução n. 466/2012. Sendo submetido, apreciado e aprovado pelo comitê de ética em pesquisa (CEP) da Universidade do Estado de Mato Grosso (UNEMAT) sob o protocolo do parecer 1.033.746।2015.

\section{RESULTADOS E DISCUSSÃO}

No presente estudo, verificou-se predominância do sexo feminino entre os profissionais atuantes da UTIN, sendo a mesma composta por profissionais entre de 20 a 35 anos, seguindo a mesma proporção entre solteiros e casados, sem filhos. Além disso, no tocante a jornada semanal de trabalho a maioria refere que seguem o máximo de 40 horas e a remuneração mensal entre 3 a 5 salários mínimos.

Os profissionais participantes da pesquisa apresentaram definições de humanização relacionando-a intrinsicamente ao processo de trabalho da enfermagem em sua prática assistencial, relacionando os meios de estabelecer conforto físico psíquico e espiritual ao paciente e à família como ações para melhora quadro clínico e satisfação dos familiares assistidos. Tal definição pode ser observada na fala de E1.

\footnotetext{
Posturas/Atitudes/Condutas realizadas no sentido de melhorar o atendimento aos neonatos/família/acompanhantes nas
}

unidades de terapia intensiva (UTI) saindo do modelo tecnocrático/instrumentalista. (E1)

A percepção da entrevistada acerca do processo de humanização em UTIN demonstra a necessidade de pensar no termo como um processo amplo, e por sua vez, complexo, abrangendo não somente os conhecimentos técnico-científicos prestados ao cliente, o neonato, mas também a incorporação de posturas que promovam as relações afetivas do binômio mãe-filho e de sua família. ${ }^{15}$ A partir deste posicionamento pode-se identificar a importância de humanizar a assistência de enfermagem em todos os momentos, estando a atenção integral e como um fator determinante para adoção de condutas que envolvam o desenvolvimento de um cuidado planejado, voltado a atender as necessidades dos familiares, imprescindível à recuperação do neonato. ${ }^{16,17}$

Todavia, buscar meios para superar o modelo "tecnocrático/ instrumentalista", volta-se à ideia de que o $\mathrm{RN}$ está exposto a vários eventos/estímulos estressantes e dolorosos durante o processo de hospitalização, causados pela rotina de procedimentos, muitas vezes invasivos presentes na terapia intensiva e que afasta a família do contato íntimo com os novos membros. A inclusão de tecnologias de 
cuidados leves, seria uma alternativa a um cuidado de enfermagem mais humanizado possibilitando a participação ativa do paciente em atividades que contribuam para $\mathrm{o}$ atendimento às suas necessidades e as de sua família. ${ }^{18}$

A participante $\mathrm{E} 4$ entende que a humanização do cuidado, deve ser ampliada e estendida à família do RN hospitalizado seguindo as mesmas definiçõos apresentadas pelas demais participantes, em sua fala traz meios que poderiam possibilitar atenção mais humanística de atenção aos pacientes.

[...] prestar informações aos familiares, de maneira clara $e$ compreensivel, tratar o paciente com um ser participante de uma sociedade, de um círculo familiar e não apenas como uma doença; compreender $e$ respeitar o momento difícil que significa para a família, aquele $R N$ estar internado, pois em sua maioria, foi uma criança muito esperada e que por ora foi separada da família e principalmente da mãe; explicar os procedimentos realizados de forma que a família compreenda; uma assistência holística. (E4)

Ao tratar sobre assistência de enfermagem holística, a participante E4 retoma a ideia de que o profissional enfermeiro deve agir de modo fundamentado, a partir das diversas dimensões do ser humano, ofertando maiores possibilidades terapêuticas de recuperação e cuidado diante do adoecimento. Desta forma, a posição que deve ser adotada pelos profissionais de saúde, devem-se basear nas necessidades expressas pelo cliente e familiares em seus contextos de sofrimento, buscando o cuidado conforme suas necessidades e respeitando as limitações. Para tanto, o desenvolvimento da empatia é fundamental, por conduzir o profissional a reflexões sobre suas condutas na assistência e as repercussões destas na saúde dos clientes. ${ }^{8}$, 19

Não obstante, a internação em uma UTIN traz impactos na rotina do bebê e de seus pais, que podem ampliar os sofrimentos de ambos. Ademais, dispor de profissionais habilitados com visões teóricas de trabalho que fogem do tecnicismo permite dirimir maiores complicações. ${ }^{17}$ Nesse sentido, o acolhimento da família contribui para a minimização de aspectos negativos, como medos, angústias e frustrações, aumentando o vínculo da família com a equipe de saúde e permitindo melhora na atenção ao RN. ${ }^{20,}$ 21

A inclusão da família no cuidado para com o RN hospitalizado é destacado por algumas participantes da pesquisa, onde relatam que para fortalecer a ação da família durante a internação o profissional de orientá-la e situá-la diante dos cuidados que 
estão sendo realizados, tornando-os integrantes do processo de cuidar e os informando quanto o ambiente novo, na maioria das vezes, que é a UTIN.

Deixo os familiares livres para verem o que estamos fazendo com sua criança. Mas, sempre vamos orientando sobre o que é e suas dúvidas. (E2)

Procuro conversar sempre com os familiares, perguntar as dúvidas, sanar as dúvidas que eu tenho possibilidade em responder, tento tranquilizar os pais em relação ao tratamento que está sendo ofertado na UTI [...] então eu tento conversar e explicar para os pais o que está acontecendo com o bebe, que muitas vezes só estão ali apenas para ganhar peso ou melhorar as condições de saúde para poder ir para casa [...]. (E4)

Percebe-se nos relatos uma preocupação por parte da equipe, partindo do entendimento da importância do envolvimento familiar no processo de humanização, em sanar os anseios e dúvidas dos familiares, resultando na maior confiabilidade dos pais na equipe e diminuindo ansiedades causadas pelo medo que o serviço de saúde intensivo proporciona. O medo das famílias com parentes em UTI deve-se ao estigma de ser um ambiente onde as pessoas estão em alto risco de morte, o que acarreta na família o sentimento de incerteza, insegurança, desespero e estresse. Assim, cabe aos profissionais de enfermagem, aproximar o binômio mãe-filho quando possível, ou paifilho, podendo assim tranquilizar a família, quebrando através do diálogo, da empatia e do esclarecimento de dúvidas, estereótipos desse ambiente de cuidado. ${ }^{22,23}$

Através da assistência humanizada prestada ao RN e sua família, os profissionais desse setor podem diminuir possíveis danos emocionais à família, estimulando a sua participação no cuidado, por intermédio do empoderamento, trazendo-os próximos aos problemas e estratégias de solução determinados, para que tenham conhecimento sobre o estado de saúde dos pequenos pacientes e entendam a necessidade de certas técnicas ou tratamentos muitas vezes invasivos. A incorporação da família como importante objeto do plano assistencial identificada nos relatos das participantes, demonstra a necessidade constante de abordagem com visão holística da categoria de enfermagem, configurando-se como uma das mais importantes para a ação promotora da humanização em UTIN graças a seu contato constante e íntimo com pacientes e familiares, tal dado também pode ser identificado nas falas a seguir. ${ }^{24,25,17}$

Todos nós fazemos, mas cada um do seu jeito, respeitamos e consideramos muito a família. (E1)

[...] trato com cuidado e respeito à família, acredito que as mesmas 
coisas se repetem entre os profissionais. (E2)

[...] nunca observei a família não ser tratada com respeito e dignidade. Eles precisam muito de nós e nos dedicamos. (E3)

Embora o saber técnico-científico da enfermagem no ambiente intensivo seja essencial para uma boa prática profissional, não há como desvincular o ser humano de sua individualidade e do seu vínculo-afetivo familiar, indispensáveis para o bom desenvolvimento psicoemocional do indivíduo. ${ }^{25,26}$ Tratando-se de humanização do ambiente, as profissionais relatam aderirem à condutas que auxiliam na terapêutica.

Manter luzes baixas, não falar muito alto, não colocar prontuários/pranchetas em cima das incubadoras, acolher a família. (E3)

O cuidado que tenho é de sempre tentar manter o ambiente o mais humanizado e tranquilo para ajudar na recuperação da mãe e do $R N$, cuidamos da iluminação, do excesso de som e de explicar a importância desses cuidados para a família. (E1)

Com base nos relatos acima, é possível evidenciar a associação do cuidado humanizado com às intervenções durante a assistência, no tocante a percepção sensorial do RN com relação ao ambiente da UTIN, tais como, o cuidado com a sonoridade e iluminação, além de ressaltarem, novamente, a família como parte do cuidado e a proteção a dignidade humana do
RN. Um estudo descreve a importância de boas práticas no cuidado com o RN para favorecer sua adaptação ao mundo e recuperação em caso de hospitalização. ${ }^{27} \mathrm{O}$ controle sobre os ruídos em UTIN são, por vezes, um viés inerente das próprias atividades assistenciais, o que torna seu controle uma tarefa difícil, por outro lado, de suma importância para o bem-estar e desenvolvimento saudável do neonato. ${ }^{28,29}$ A iluminação tem importante papel na promoção do conforto ao neonato, devendo sempre se manter amena. Uma possibilidade, encontrada na literatura, quando há meios para redução da luz durante alguns períodos do dia é utilizar o "tetinho", que consiste na sobreposição de um lençol sob a incubadora de forma a diminuir a incidência de luminosidade no neonato, tal técnica tem demonstrado bons efeitos no conforto e diminuição de estresse dos pequenos pacientes. ${ }^{29,30}$

$$
\text { É importante o olhar do }
$$
profissional sobre o ser humano que está sob seus cuidados, que embora frágil e indefeso ainda é um indivíduo, devendo o cuidado ter uma abordagem centrada da manutenção da dignidade humana e na integralidade, fugindo da prática rotineira e mecanicista. ${ }^{28}$ Quando os profissionais deste estudo foram questionados sobre o quão humanizado era o ambiente em que 
trabalhavam, em sua maioria afirmam que a estrutura impede o desenvolvimento de algumas práticas de humanização, mas que consideram a assistência humanizada, graças ao cuidado da equipe de enfermagem com os neonatos e seus familiares.

Sim, devido o respeito, o carinho e atenção da equipe. Poderia ser mais humanizado, mas talvez a estrutura ofertada pelo hospital não faz isso se tornar possível. Porém, a equipe faz o que pode, dentro das suas possibilidades. (E4)

A humanização é um desafio, fazemos nosso melhor, mas não é padronizado, institucionalizado [...], trazemos o aprendizado da faculdade e vamos melhorando no dia a dia. (E2)

Estudos

desenvolvidos

corroboram com dificuldades apresentadas pelas participantes, apontando como empecilhos na implementação das estratégias propostas, fatores como a precária estrutura física das unidades, a falta de educação continuada com os profissionais e a pouca relevância dada a matéria pela gestão e chefias dos serviços. ${ }^{31}$, ${ }^{6}$ Dessa forma, observa-se que a prestação do cuidar humanizado é uma prioridade, mesmo que, por vezes, as ações se esbarrem em limitações que lhes são impostas, entretanto os profissionais tem conhecimento sobre a necessidade e a obrigatoriedade de atender humanamente seus pacientes, esforçando-se para ofertar o máximo de conforto possível e o mínimo de riscos. ${ }^{31,32,33}$

A UTIN gera ansiedade para pacientes, família e profissionais, a relação do cansaço relacionado a exigência do saber técnico, a carga de trabalho neste local, as questões de vida e morte iminentes aliados as questões éticas geram uma sobrecarga nos profissionais atuantes neste ambiente, $o$ que pode fazer com que algumas ações humanizadas que despendem certo tempo não sejam realizadas. ${ }^{33}$ Desta maneira as emoções são potencializadas na UTIN, envolvendo desde a família até os profissionais de saúde, estes que sensibilizados buscam ampliar a qualidade do serviço prestado e da humanização, porém podem apresentar vulnerabilidades que consequentemente podem afetar a assistência. ${ }^{34}$

\section{CONSIDERAÇÕES FINAIS}

O conceito de humanização vem sendo bastante difundido desde o início dos anos 2000, e deve ser amplamente discutido desde a academia até os serviços de saúde em seus diferentes níveis de complexidade, devido à amplitude do tema e as subjetividades embutidas no conceito. Quando se trata da prestação do cuidado intensivo à um usuário considerado tão frágil e vulnerável como um $\mathrm{RN}$, o tema 
humanização perpassa a técnica excelente e adentra no campo das emoções, tanto dos próprios profissionais quanto dos familiares, do acolhimento, melhorias das estruturas/ambientes da UTIN, dos processos e das condições de trabalho.

No presente estudo observou-se que os profissionais demonstram grande conhecimento sobre a humanização, compreendendo a mesma como um processo vivencial, que é adquirido por intermédio da experiência da prática clínica sob um viés afetivo e da própria ciência da enfermagem. Ainda ressaltaram a importância da sua prática na prestação do cuidado de enfermagem ao neonato, devendo a atenção humanizada ser estendida à família do RN hospitalizado, buscando fortalecer os vínculos do binômio mãe-filho.

Além disso, percebeu-se uma preocupação dos profissionais, identificando a importância do envolvimento familiar no processo de humanização, quando buscam esclarecer todas as dúvidas demandadas pelos pais, o que gera a confiança destes na equipe e diminui anseios gerados pelo ambiente e intervenções invasivas. Destaca-se ainda, o processo de empoderamento gerado nos pais pela equipe, quando estimulam a participação dos mesmos nos cuidados prestados ao RN.

A limitação do estudo refere-se ao fato de terem sido investigados somente os profissionais enfermeiros, excluindo-se os outros profissionais que integram a equipe multiprofissional. Assim, novas investigações sobre a percepção de outros profissionais em relação à humanização da assistência são de grande valia no campo da saúde e, especialmente da atenção intensiva em neonatologia, desafio inter e transdisciplinar.

\section{REFERÊNCIAS}

1. Santos, AAS. Humanização em UTI neonatal: análise da literatura sobre a atuação da enfermagem na tríade mãe, recém-nascido [Internet]. [monografia]. Florianópolis: Universidade Federal de Santa Catarina; 2014. [citado em 06 maio 2017]. Disponível em: https://repositorio.ufsc.br/handle/12345678 9/171882

2. Ministério da Saúde (Brasil). Secretaria de Políticas de Saúde, Área de Saúde da Criança. Atenção humanizada ao recémnascido de baixo peso: método mãecanguru: manual do curso [Internet]. Brasília, DF: Ministério da Saúde; 2002 [citado em 30 jul 2018]. 282 p. (Série A. Normas e Manuais Técnicos; n. 145). Disponível em: http://www.redeblh.fiocruz.br/media/manu alcanguru.pdf

3. Oliveira SJGS, Melo SN, Oliveira SMB, Sousa DS, Pinheiro FGMS. Assistência humanizada no período perinatal com a utilização do método canguru: uma revisão bibliográfica. Cad Grad Ciênc Biol Saúde 
[Internet]. out 2014 [citado em 06 maio 2017]; 2(2):79-91. Disponível em: https://periodicos.set.edu.br/index.php/cad ernobiologicas/article/view/1480/1014 4. Silva AR, Hofmann E., Zancaron SS. Acolhimento na Unidade de Terapia Intensiva Neonatal: percepções das profissionais e mães. Argum. [Internet]. 2018 [citado em 30 jul 2018]; 10(1):198212. Disponível em: https://periodicos.ufes.br/argumentum/artic le/view/18739/13201

5. Reis LS, Silva EF, Waterkemper R, Lorenzini E, CecChetto FH. Percepção da equipe de enfermagem sobre humanização em unidade de tratamento intensivo neonatal e pediátrica. Rev Gaúch Enferm. [Internet]. 2013 [citado em 30 jul 2018]; 34(2):118-24. Disponível em: https://www.scielo.br/pdf/rgenf/v34n2/v34 n2a15.pdf

6. Fialho FA, Dias IMAV, Santos RS, Silva LR, Salvador M. Humanização permeando o cuidado de enfermagem neonatal. Rev Enferm UFPE on line. [Internet]. 2016 [citado em 06 maio 2017]; 10(7):2412-19. Disponível em: https://periodicos.ufpe.br/revistas/revistaen fermagem/article/view/11297/12960 7. Cotta RMM, Reis RS, Campos AAO, Gomes AP, Antonio VE, Siqueira-Batista $R$. Debates atuais em humanização e saúde: quem somos nós? Ciênc Saúde Colet. [Internet]. 2013 [citado em 30 jul 2018]; 18(1): 171-79. Disponível em: https://www.scielo.br/pdf/csc/v18n1/18.pdf 8. Oliveira K, Veronez M, Igarashi IH, Corrêa DAM. Vivências de familiares no processo de nascimento e internação de seus filhos em UTI neonatal. Esc Anna Nery Rev Enferm. [Internet]. 2013 [citado em 30 jul 2018]; 17(1):46-53. Disponível em: https://www.scielo.br/pdf/ean/v17n1/07.pd f

9. Buffoli M, Bellini E, Bellagarda A, Di Noia M, Nickolova M, Capolong S. Listening to people to cure people: the
LpCp - tool, an instrument to evaluate hospital humanization. Ann Ig. [Internet]. 2014 [citado em 30 jul 2018]; 26(5):44755. doi: 10.7416/ai.2014.2004. 10. Rocha MCP, Carvalho MSM, Fossa AM, Rossato LM. Assistência humanizada na terapia intensiva neonatal: ações e limitações do enfermeiro. Saúde Rev. [Internet]. 2015 [citado em 30 jul 2018]; 15(40):67-84. Disponível em: https://www.metodista.br/revistas/revistasunimep/index.php/sr/article/view/2534/147 6

11. Medeiros AC, Siqueira $\mathrm{HCH}$, Zamberlan C, Cecagno D, Nunes SS, Thurow MRB. Comprehensiveness and humanization of nursing care management in the Intensive Care Unit. Rev Esc Enferm USP. [Internet]. 2016 [citado em 17 maio 2017]; 50(5):816-22. Disponível em: https://www.scielo.br/pdf/reeusp/v50n5/00 80-6234-reeusp-50-05-0817.pdf

12. Nascimento VF, Maciel MM, Lemes AG, Borges AP, Terças ACP, Hattori TY. Percepções de familiares sobre hospitalização no ambiente intensivo. Rev Enferm UFPI. [Internet]. 2015 [citado em 30 Jul 2018]; 4(2):92-9. Disponível em: https://revistas.ufpi.br/index.php/reufpi/arti cle/view/3402/pdf

13. Bardin L. Análise de conteúdo. 70ed. Lisboa: Edições 70, 2010. 280 p. 14. Fontanella BJB, Luchesi BM, Saidel MGB, Ricas J, Turato ER, Melo DG. Amostragem em pesquisas qualitativas: proposta de procedimentos para constatar saturação teórica. Cad Saúde Publica [Internet]. 2011 [citado em 30 jul 2018]; 27(2):389-94. Disponível em: https://www.scielo.br/pdf/csp/v27n2/20.pd $\mathrm{f}$

15. Passos SSS, Silva JO, Santana VS, Santos VMN, Pereira A, Santos LM. O acolhimento no cuidado à família numa unidade de terapia intensiva. Rev Enferm UERJ. [Internet]. 2015 [citado em 30 jul 2018]; 23(3):368-74. Disponível em: https://www.e- 
publicacoes.uerj.br/index.php/enfermagem uerj/article/view/6259/13776

16. Almeida CR, Morais AC, Lima KDF, Silva ACOC. Cotidiano de mães acompanhantes na unidade de terapia intensiva neonatal. Rev Enferm UFPE on line [Internet]. 2018 [citado em 30 jul 2018]; 12(7):1949-56. Disponível em: https://periodicos.ufpe.br/revistas/revistaen fermagem/article/view/22640/29523 17. Nascimento VF, Silva RCR. Assistência de enfermagem ao recémnascido pré-termo frente às possíveis intercorrências. Rev Enferm UFSM. [Internet]. 2014 [citado em 30 jul 2018]; 4(2):429-38. Disponível em: https://periodicos.ufsm.br/reufsm/article/vi ew/10252/pdf

18. Santos LC, Vorcaro AMR. Implicações da patologia e da hospitalização do bebê ao nascer: a contribuição da psicanálise e de seu método clínico. Estilos Clin. [Internet]. 2016 [citado em 17 maio 2017]; 21(2):282301. Disponível em http://pepsic.bvsalud.org/scielo.php?script =sci_arttext\&pid=S1415-

$71282016000200002 \& \operatorname{lng}=$ pt\&nrm=iso 19. Sá Neto JA, Rodrigues BMRD. A ação intencional da equipe de enfermagem ao cuidar do RN na UTI neonatal. Ciênc Cuid Saúde [Internet]. 2015 [citado em 31 jul 2018]; 14(3):1237-44. Disponível em: http://periodicos.uem.br/ojs/index.php/Cie ncCuidSaude/article/view/22320/15383 20. Pereira RMS, Câmara TL, Pereira NCST. Enfermagem e o manuseio do recém nascido na Unidade de Terapia Intensiva Neonatal. Rev Uningá [Internet]. 2019 [citado em 31 jul 2018]; 56(S2):22233. Disponível em: http://revista.uninga.br/index.php/uninga/ar ticle/view/2156/1915

21. Salcedo EAC, Freston YMB, Souza JLE, Costa SM, Duarte CM, Batista PM, et al. Experiência de cuidados e humanização em uti neonatal - grupo de pais enlutados. In: II SIMTEC:
Simpósio de Profissionais da Unicamp [Internet]; 2008; Campinas. Campinas: UNICAMP; 2008 [citado em 31 jul 2018]. p. 271. Disponível em:

https://econtents.bc.unicamp.br/inpec/inde x.php/simtec/article/view/8721/4114 22. Rocha SS, Olivindo DDF, Sá CN, Fonseca LF. Percepção da enfermagem em relação às mães no cuidado de recémnascidos na unidade de terapia intensiva neonatal. Enferm Foco (Brasília)

[Internet]. 2013 [citado em 31 jul 2018]; 4(1):45-8. Disponível em:

http://revista.cofen.gov.br/index.php/enfer magem/article/view/502/192

23. Montes LAB, Prieto AM. Uncertainty associated to parents of preterm infants hospitalized in neonatal intensive care units. Invest Educ Enferm. [Internet]. 2016 Jun [citado em 20 fev 2018]; 34(2):360-7. doi: 10.1590/S0120-53072016000200016 24. Magalhães SGS, Silva JSLG. O cuidado humanizado na Unidade de Terapia Intensiva Neonatal. Rev PróUniverSUS [Internet]. 2019 [citado em 30 jul 2018]; 10(1):129-32. Disponível em: http://editora.universidadedevassouras .edu.br/index.php/RPU/article/view/1640 25. Rocha DKL, Ferreira HC. Estado da arte sobre o cuidar em neonatologia: compromisso da enfermagem com a humanização na unidade de terapia intensiva neonatal. Enferm Foco (Brasília). [Internet]. 2013 [citado em 31 jul 2018]; 4(1):24-8. Disponível em: http://revista.cofen.gov.br/index.php/enfer magem/article/viewFile/497/187

26. Lopes LCA. Boas práticas no cuidado ao recém-nascido: construção de um guia voltado para a prática [Internet]. [trabalho de conclusão de curso]. Florianopólis: Universidade Federal de Santa Catarina; 2017 [citado em 03 ago 2018]. Disponível em:

https://repositorio.ufsc.br/xmlui/handle/12 3456789/172892

27. Moretto LCA, Perondi ER, Trevisan MG, Teixeira GT, Hoesel TC, Costa LD. 
Dor no recém-nascido: perspectivas da equipe multiprofissional na unidade de terapia intensiva neonatal. Arq Ciências Saúde UNIPAR.. [Internet]. 2019 [citado em 03 ago 2018]; 23(1):29-34. Disponível em:

https://www.revistas.unipar.br/index.php/s aude/article/view/6580

28. Santos BR, Orsi KCSC, Balieiro

MMFG, Sato MH, Kakehashi TY, Pinheiro EM. Efeito do "horário do soninho" para redução de ruído na unidade de terapia intensiva neonatal. Esc. Anna Nery Rev. Enferm. [Internet]. 2015 [citado em 05 jan 2018]; 19(1):102-6. Disponível em: https://www.scielo.br/pdf/ean/v19n1/14148145-ean-19-01-0102.pdf

29. Jordão KR, Pinto LAP, Machado LM, Costa LBVL, Trajano ETL. Possíveis fatores estressantes na unidade de terapia intensiva neonatal em hospital universitário. Rev Bras Ter Intensiva. [Internet]. 2016 [citado em 03 ago 2018]; 28(3):310-14. Disponível em: https://www.scielo.br/pdf/rbti/v28n3/0103507X-rbti-20160041.pdf

30. Reis LS, Silva EF, Waterkemper R, Lorenzini E, Cecchetto FH. Percepção da equipe de enfermagem sobre humanização em unidade de tratamento intensivo neonatal e pediátrica. Rev Gaúch Enferm. [Internet]. 2013 [citado em 03 ago 2018]; 34(2):118-24. Disponível em: https://www.scielo.br/pdf/rgenf/v34n2/v34 n2a15.pdf

31. Mongiovi VG, Anjos RCCBL, Soares SBH, Lago-Falcão TM. Reflexões conceituais sobre humanização da saúde: concepção de enfermeiros de Unidades de Terapia Intensiva. Rev Bras Enferm. [Internet]. 2014 [citado em 03 ago 2018]; 67(2):306-11. Disponível em: https://www.scielo.br/pdf/reben/v67n2/003 4-7167-reben-67-02-0306.pdf 32. Roseiro CP, Paula KMP. Concepções de humanização de profissionais em Unidades de Terapia Intensiva Neonatal. Estud Psicol. (Campinas) [Internet]. 2015 [citado em 03 ago 2018]; 32(1):109-19. Disponível em:

https://www.scielo.br/pdf/estpsi/v32n1/010 3-166X-estpsi-32-01-00109.pdf

33. Silva SB. Campanha do silêncio na UTI neonatal da Maternidade Nossa Senhora de Nazareth em Boa Vista - RR [Internet]. [trabalho de conclusão de curso]. Boa Vista: Universidade Federal de Santa Catarina; 2014 [citado em 03 ago 2018]. Disponível em: https://repositorio.ufsc.br/xmlui/handle/12 3456789/173225

34. Loudet CI, Marchena MC, Maradeo MR, Fernández SL, Romero MV, Valenzuela GE et al. Diminuição das úlceras por pressão em pacientes com ventilação mecânica aguda prolongada: um estudo quase-experimental. Rev Bras Ter Intensiva [Internet]. 2017 [citado em 03 ago 2018]; 29(1):39-46. Disponível em: https://www.scielo.br/pdf/rbti/v29n1/0103507X-rbti-29-01-0039.pdf

RECEBIDO: 04/04/2019

APROVADO: 09/04/2020 PUBLICADO: 07/2020 